\title{
Memory scanning of young and old adults: The influence of rate of presentation and delay interval on recognition memory performance
}

\author{
CHARLES I. MANISCALCO and DONALD V. DeROSA \\ Bowling Green State University, Bowling Green, Ohio 43403
}

\begin{abstract}
The present study investigated memory scanning of 12 young (mean age $=19.0$ years) and 12 old (mean age $=67.7$ years) subjects. Reaction times were measured in a recognition memory task developed by Sternberg (1966), using a variety of presentation conditions. The mean scanning rates for young and old subjects were similar to those obtained in previous research when slow rates of presentation were used. However, with rapid rates of presentation, older subjects do just as well as young subjects. An examination of individual subjects' data indicated that $\theta$ of the 12 old subjects scanned at much the same rate as the young subjects even when slow rates were used, whereas the remaining 3 subjects scanned much more slowly than all others in both age groups.
\end{abstract}

Sternberg (1966) has developed a paradigm for studying recognition memory processes using reaction time (RT). In his study, subjects were shown a series of from two to four to-be-remembered items (digits) called the positive set, followed by a test stimulus (probe), to which they were to make a positive response if the probe was contained in the list, and a negative response otherwise. It was found that RT increased linearly as a function of the size of the positive set for both positive and negative probes, with the slopes of the two functions being essentially equal ( $35-40 \mathrm{msec} / \mathrm{unit}$ increase in set size).

Sternberg (1966) argued that these results support a serial exhaustive model of memory search in which an internal representation of the probe item is compared successively to each member of the positive set, resulting in either a match or a mismatch. The serial nature of the search process is supported by the fact that the RT function increases linearly with increases in set size. The exhaustive aspect of the search process is evidenced by the finding that the slopes of the functions for positive and negative responses are equal.

Recently, this experimental paradigm has been used to investigate the effects of aging on short-term and long-term recognition memory (Anders \& Fozard, 1973; Anders, Fozard, \& Lillyquist, 1972; Eriksen, Hamlin, \& Daye, 1973). Anders et al. (1972), using digits and positive-set sizes of one, three, five, and seven items, found that the search rate for old subjects $(71 \mathrm{msec} /$ item; mean age $=68.1$ years) was significantly slower than that for young subjects $(39 \mathrm{msec} /$ item; mean age $=$

The authors would like to express their appreciation to Kirk Smith, whose comments and suggestions on the manuscript were most helpful.
20.0 years). Anders and Fozard (1973) successfully replicated and extended these findings using a single positive set followed by a number of probe items (fixedset procedure). All of these researchers conclude that there is a basic slowing of mental processes with age, resulting in slower search rate.

There remains an unanswered question: Why do old subjects scan more slowly than young subjects? One possibility is that the encoded stimuli for old subjects are of poorer quality than those for young subjects. That is, when the positive set was presented only moments before the probe (varied-set procedure), the increased search rates for old subjects may have been due to incomplete encoding of the positive-set items. Also, in either the fixed-set or varied-set procedure, the memorial representation of the probe item may have been poorer for the old subjects. In fact, Sternberg (1969) reported a study in which probe items were partially degraded, resulting in a search rate slower than that obtained for intact probes. If old subjects have relatively greater difficulty in encoding the probe or encoding the positive-set items, slower search rates may result.

Two studies lend indirect support to the possibility that encoding processes become slower with age. Eriksen, Hamlin, and Breitmeyer (1970) found that old subjects (50-55 years old) required longer exposure durations than young subjects (30-35 years old) in order to identify forms. Also, Welsandt, Zupnick, and Meyer (1973), in a backward visual masking study, concluded that older subjects (55 years old) extracted information from the icon more slowly than young subjects (1935 years old). The use of the same presentation rate and delay interval for young and old subjects, then, may differentially handicap the old subjects. Consequently, the present study varied the presentation rate and delay 
interval in an effort to determine whether encoding differences exist between young and old subjects and to assess the effects of these variables on recognition memory performance.

\section{METHOD}

\section{Subjects}

The young subjects were four male and eight female undergraduate students (mean age $=19.0$ years, ranging from 18.4 to 20.6 years; mean education $=12.23$ years) enrolled in an introductory psychology course at Bowling Green State University. The old subjects were three male and nine female volunteers from the northwest Ohio area (mean age $=67.6$ years, ranging from 64.7 to 73.5 years; mean education $=16.67$ years). Both groups were paid for their participation. The old subjects represent a highly educated group. All had undergraduate degrees, and seven had at least some graduate work. Although nine were retired, virtually all of these subjects led active lives. These subjects can be contrasted with those used by Anders and Fozard (1973) and Anders et al. (1972), who were male veterans.

\section{Stimuli and Apparatus}

Subjects were individually tested in a sound-deadened isolation chamber, with the digits 1-9 presented visually on an I.E.E. display cell mounted $3 \mathrm{ft}$ from the subjects. The subjects' RTs were measured to the nearest $.001 \mathrm{sec}$ and were recorded on punched paper tape for summary by computer. Accuracy feedback was automatically provided after each trial.

\section{Procedure}

Each trial, preceded by a warning signal, consisted of a serial presentation of three, four, or five digits (positive set), followed by another warning signal (an amber light centered directly above the display cell), and then a single digit (probe). Two presentation rates were used: (1) fast-each element of the positive set on for $.3 \mathrm{sec}$, with a .3 -sec off-time between elements $(1 \mathrm{item} / .6 \mathrm{sec})$, and (2) slow-1-sec on-time and offtime for each element $(1 \mathrm{item} / 2 \mathrm{sec})$. There were two delay intervals between the last item of the positive set and the probe item: (1) brief-.7-sec delay, and (2) long-3.0-sec delay. Subjects were instructed to depress a key marked "yes" if the probe was identical to any member of the positive set and to depress a key marked "no" otherwise. Both speed and accuracy were emphasized in the instructions.

\section{Experimental Conditions}

Subjects received a series of practice trials and were shown 380 experimental trials during a 1.5-h experimental session. The positive sets were constructed through random selection without replacement of the digits $1-9$, with runs of consecutive digits no greater than two. The combination of presentation rates and delay intervals yielded four conditions: (1) fast presentation/ brief delay interval (fast-brief), (2) fast presentation rate/long delay interval (fast-long), (3) slow presentation rate/brief delay interval (slow-brief), and (4) slow presentation rate/long delay interval (slow-long). Presentation of the trials was blocked according to condition (95 trials/block), with the order of the four blocks of trials counterbalanced across all subjects. Rest periods were provided after each block of trials. Probe items were selected from each serial position (SP) in the positive set with equal frequency, and the probability, over trials, of a positive probe was .5 .

\section{RESULTS AND DISCUSSION}

\section{Reaction Time Data}

Mean RTs for correct responses were computed for

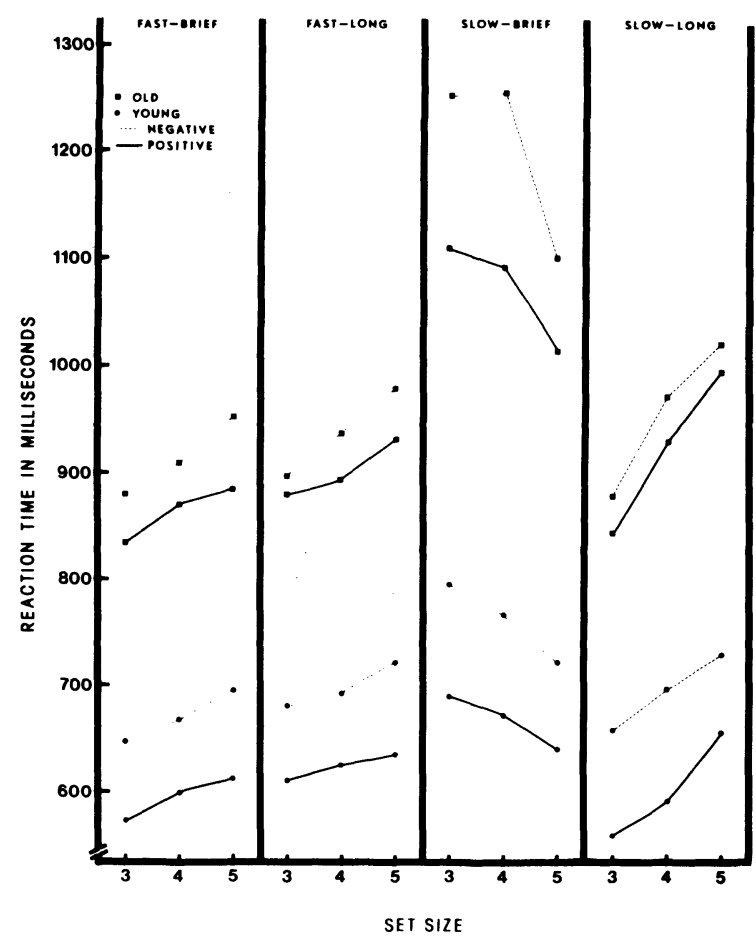

Figure 1. Reaction time in milliseconds as a function of set size for old and young adults (each panel depicts a presentation condition).

each subject individually. The error rates were low, both for young subjects (mean $=4.5 \%$, ranging from $.5 \%$ to $11.1 \%$ ) and for old subjects (mean $=5.0 \%$, ranging from $.8 \%$ to $11.1 \%$ ). In Figure 1, set-size functions are shown for each presentation rate and delay interval combination. It can be seen that for three of the four conditions, the typical increasing set-size functions were observed. For the slow-brief condition (third panel), however, the set-size functions had negative slopes for both age groups, perhaps suggesting a search of the negative set. Two findings suggest an alternative explanation. First, given a search of the negative set, the SP of the probe item in the positive set should not affect RT. However, for the slow-brief condition (and for all other conditions), there was a marked recency effect, with RT decreasing with increasing SP. These findings strongly suggest that the search is conducted through the positiveset items, and not through the items in the negative set. Second, many subjects reported that, for the slow-brief condition, they were surprised and unprepared when the probe item appeared after three or four positive-set items. It appears that subjects were preparing to receive another slowly presented positive-set item and were relatively unprepared for the appearance of the probe. Consequently, the RTs for Set Sizes 3 and 4 were elevated. Since subjects were aware that Set Size 5 was the longest one used, no uncertainty, and thus, no elevation of RT, occurred. Given that the slow-brief condition yielded results qualitatively different from those for the other 
conditions, and since these results do not permit an examination of search rates, these data were omitted from the analyses on the overall data.

\section{Overall Analyses}

An analysis of variance was conducted on the mean RTs (omitting the slow-brief condition), with one between-subjects factor, age, and three within-subjects factors, set size (three, four, and five), condition (fastbrief, fast-long, and slow-long), and response type (positive and negative). The results of this analysis indicate that the set-size effect was significant $[F(2,44)$ $=47.64, \mathrm{p}<.001]$, and when orthogonally decomposed, only the linear component of the set-size variable was significant $[F(1,22)=60.22, p<.001]$. This linear set-size effect suggests a serial search process. Although negative responses took longer than positive responses $[F(1,22)=16.09, p<.001]$, a lack of a significant Set Size by Response Type interaction supports an exhaustive model of memory scanning.

It can be seen in Figure 1 that old subjects had longer overall latencies than young subjects $[F(1,22)=15.91$, $\mathrm{p}<.001]$, replicating earlier findings. Neither the condition effect nor the Age by Condition interaction was significant, indicating that, within each age group, the overall latencies were not different for the three conditions. However, the linear component of set size did interact with condition $[\mathrm{F}(2,44)=8.86, \mathrm{p}<.001]$. An orthogonal decomposition indicated that this interaction effect was due to the slow-long condition's yielding a larger linear set-size effect (slower scanning rates) than the combination of the other two conditions $[F(1,22)=16.89, p<.001]$. This result was surprising. It was expected that allowing more presentation time for individual items and a longer delay interval before presentation of the probe would facilitate a rapid search, especially for subjects in the old group. That is, if the fast presentation rates resulted in poorly encoded stimuli for old subjects, allowing more presentation time per item should improve the quality of the encoded stimuli and result in faster search rates. However, for old and young subjects, the relatively slow presentation of the stimuli resulted in slower search rates. Many subjects in both groups reported that the slow rates in the slow-long condition had the effect of reducing their alertness, sometimes to the point of boredom. The slower search rates, then, may be due to changes in attentional or motivational factors. Nevertheless, the observed increased search rates and the subjects' introspective reports are clearly inconsistent with the notion of encoding deficits for old subjects.

The critical question of whether young and old subjects scan at different rates can be answered by examining the Age by Set Size interaction. In the present study, this interaction approached but did not achieve significance $[F(2,44)=3.16, p>.05 ;$ critical $F=3.21]$. The interaction of age with the linear component of set size also failed to reach significance $[F(1,22)=3.81$, $p>.05$; critical $F=4.30]$. Thus, there are no clear age differences in rate of scanning in the present investigation.

A nonparametric signal detection analysis (Grier, 1971) was applied to the data in an added attempt to determine if age differences existed in the quality of the encoded stimuli or in cautiousness. No such differences were found.

\section{Comparison of Results With Previous Research}

In an effort to further explore the possibility of agerelated scanning rate differences, Table 1 presents a comparison of the results of the present study with those of previous studies using a varied-set procedure. Examining the ratio of intercepts for old subjects to those for young subjects, as well as the absolute differences, it can be seen that the results are quite similar across all studies. Turning to the slope comparisons, it can be seen that for the slow-long condition, the ratio is similar to the other studies. However, for the fast-brief and fast-long conditions, both the ratios and absolute differences are less than those resulting from similar procedures. Thus, only for one of the three conditions do the slope ratios and differences compare closely with those from previous research.

\section{Individual Subject Data}

The lack of a significant Age by Set Size interaction for the overall analysis is still difficult to interpret,

Table 1

Comparison of Results With Those From Previous Research

\begin{tabular}{|c|c|c|c|c|c|c|c|c|}
\hline \multirow[b]{3}{*}{ Study } & \multicolumn{4}{|c|}{ Best-Fitting Lines } & & & & \\
\hline & \multicolumn{2}{|c|}{ Young } & \multicolumn{2}{|c|}{ Old } & \multicolumn{2}{|c|}{ Slope Ratio } & \multicolumn{2}{|c|}{ Intercept Ratio } \\
\hline & RT & $\mathbf{V}$ & RT & $\mathbf{V}$ & $0 / Y$ & D & O/Y & $\mathbf{D}$ \\
\hline Anders et al. (1972) & $623+39 N$ & 98 & $816+71 N$ & 98 & 1.82 & 32 & 1.31 & 193 \\
\hline Anders \& Fozard (1973) & $680+23 N$ & 98 & $892+45 N$ & 98 & 1.96 & 22 & 1.31 & 212 \\
\hline $\begin{array}{l}\text { Maniscalco \& DeRosa } \\
\text { Fast-Brief } \\
\text { Fast-Long } \\
\text { Slow-Long } \\
\end{array}$ & $\begin{array}{l}542+22 \mathrm{~N} \\
572+21 \mathrm{~N} \\
481+42 \mathrm{~N} \\
\end{array}$ & $\begin{array}{l}99 \\
99 \\
99\end{array}$ & $\begin{array}{l}765+30 \mathrm{~N} \\
791+32 \mathrm{~N} \\
652+72 \mathrm{~N} \\
\end{array}$ & $\begin{array}{l}99 \\
99 \\
98\end{array}$ & $\begin{array}{l}1.36 \\
1.52 \\
1.71\end{array}$ & $\begin{array}{r}8 \\
11 \\
3\end{array}$ & $\begin{array}{l}1.41 \\
1.38 \\
1.36\end{array}$ & $\begin{array}{l}223 \\
219 \\
171\end{array}$ \\
\hline
\end{tabular}

Note $-R T=$ best-fitting lines, collapsed across positive and negative responses; $V=$ variance accounted for by the best-fitting lines (in percentages). $O / Y=$ ratio of the value for old subjects to the value for young subjects; $D=$ absolute differences. 
Table 2

Individual Subjects' Slopes and Intercepts From Best-Fitting Lines

\begin{tabular}{|c|c|c|c|}
\hline \multicolumn{2}{|c|}{ Young Subjects } & \multicolumn{2}{|c|}{ Old Subjects } \\
\hline Slope & Intercept & Slope & Intercept \\
\hline 38 & 471 & 91 & 921 \\
\hline 36 & 407 & 90 & 661 \\
\hline 35 & 521 & 87 & 804 \\
\hline 34 & 895 & 49 & 661 \\
\hline 25 & 593 & 43 & 605 \\
\hline 25 & 371 & 38 & 637 \\
\hline 23 & 580 & 38 & 707 \\
\hline 21 & 583 & 27 & 744 \\
\hline 20 & 720 & 23 & 723 \\
\hline 16 & 447 & 22 & 970 \\
\hline 11 & 486 & 19 & 816 \\
\hline 9 & 539 & 14 & 586 \\
\hline
\end{tabular}

Note-Slopes are arranged in descending order. Slopes are calculated over all conditions, omitting slow-brief.

especially given that the overall slopes for young $(24 \mathrm{msec})$ and old $(43 \mathrm{msec})$ subjects are quite similar to those found by Anders and Fozard (1973) (27 and $43 \mathrm{msec}$ for young and old subjects, respectively). In their study, the Age by Set Size interaction was significant, and with fewer subjects (eight per group). In an effort to clarify this difficulty, best-fitting lines were computed for each subject, individually. The individual subjects' slopes and intercepts are presented in Table 2. For the old subjects, it can be seen that the slopes for three subjects (top three in the table) are almost twice as large as the largest in the remaining group of old subjects. Omitting these three subjects, the distributions of slopes for the two groups of subjects overlap almost completely. The Age by Set Size interaction, which approaches significance, seems to be due in large measure to 3 of the 12 old subjects.

When the three subjects with the very high slope values are omitted, the average slope drops from $43 \mathrm{msec}$ to $30 \mathrm{msec} / \mathrm{unit}$ increase in set size, thereby greatly diminishing the rather large slope difference in the two groups. Thus, it seems that the older group of subjects in the present study represent a heterogeneous group, three members of which scan much more slowly than all other subjects in the investigation. Attempts to distinguish the three slow scanners from the remaining subjects on any demographic or personal variable unfortunately all failed.

An added feature of the results indicated that the old subjects were especially handicapped in the condition in which slow rate of presentation and long retention interval are used. In fact, for 11 of the 12 old subjects, the slope in the slow-long condition was greater than the average slope for the two fast presentation conditions. To date, we are aware of no other study that used presentation rates comparable to the fast rates in the present study. Since presentation rates may influence age differences in scanning, investigators should be cautious in generalizing beyond their experimental procedures.

\section{CONCLUSIONS}

The absence of clear age-related differences in mean scanning rates points to a potentially serious problem in the use of chronological age alone as a variable in the study of the aging process. While the average results obtained in this study were comparable to those observed in similar investigations, the high degree of similarity between old and young subjects is noteworthy. The individual subject data point to the need for acknowledging the heterogeneity of the elderly and, consequently, to the inadequacy of age as an independent variable. In the future, studies should be designed to examine individual subject data and should attempt to determine those variables that are related to the speed of memory scanning. By focusing on individual subjects and on the relationship of memory scanning processes with other variables, a clear understanding of age-related differences in recognition memory performance may emerge.

\section{REFERENCES}

Anders, T. R., \& Fozard, J. L. Effects of age upon retrieval from primary and secondary memory. Developmental Psychology, 1973, 9, 411-415.

Anders, T. R., Fozard, J. L., \& Lillyquist, T. D. Effects of age upon retrieval from short-term memory. Developmental Psychology, 1972, 6, 214-217.

Eriksen, C. W., Hamlin, R. M., \& Breitmeyer, R. G. Temporal factors in visual perception as related to aging. Perception \& Psychophysics, 1970, 7, 354-356.

Eriksen, C. W., Hamlin, R. M., \& Daye, C. Aging adults and rate of memory scan. Bulletin of the Psychonomic Society, 1973, $1,259-260$

Grier, J. B. Nonparametric indexes for sensitivity and bias: Computing formulas. Psychological Bulletin, 1971, 75, 424-429.

STERNBERG, S. High-speed scanning in human memory. Science, $1966,153,652-654$.

STE Rnbera, S. Memory scanning: Mental processes revealed by reaction-time experiments. American Scientist, 1969, 57, 421-457.

Welsandt, R. F., Zupnick, J. J., \& MeYer, P. A. Age effects in backward visual masking (Crawford paradigm). Journal of Experimental Child Psychology, 1973, 15, 454-461.

(Received for publication September 9, 1982.) 